\title{
Optimized stochastic approach for integral equations
}

Venelin Todorov

Bulgarian Academy of Sciences

Institute of Mathematics and Informatics

ul. G. Bonchev 8, 1113 Sofia, Bulgaria

Bulgarian Academy of Sciences

Institute of Information and Communication Technologies

ul. G. Bonchev 25A, 1113 Sofia, Bulgaria

Email: vtodorov@math.bas.bg,venelin@parallel.bas.bg

Ivan Dimov

Bulgarian Academy of Sciences

Institute of Information and Communication Technologies

ul. G. Bonchev 25A, 1113 Sofia, Bulgria

Email: ivdimov@bas.bg

\author{
Stefka Fidanova \\ Bulgarian Academy of Sciences \\ Institute of Information and Communication Technologies \\ ul. G. Bonchev 25A, 1113 Sofia, Bulgria \\ Email: stefka@parallel.bas.bg
}

\begin{abstract}
An optimized Monte Carlo approach (OPTIMIZED MC) for a Fredholm integral equations of the second kind is presented and discussed in the present paper. Numerical examples and results are discussed and MC algorithms with various initial and transition probabilities are compared.
\end{abstract}

\section{INTRODUCTION}

$I^{2}$ NTEGRAL equations are of high importancy in various areas of applied mathematics [7]. That is why it is important to construct effective methods to solve integral equations. An important advantage of Monte Carlo (MC) methods is that they allow to search an unknown linear functional of the solution directly [1].

\section{Formulation OF THE PROBLEM}

The Fredholm integral equation of the second kind has been analyzed:

$$
u(x)=\int_{\Omega} k\left(x, x^{\prime}\right) u\left(x^{\prime}\right) d x^{\prime}+f(x) \text { or } u=\mathcal{K} u+f,
$$

where

$x, x^{\prime} \in \Omega \subset \mathbb{R}^{d}, u(x), f(x) \in L_{2}(\Omega), k\left(x, x^{\prime}\right) \in L_{2}(\Omega \times \Omega)$

and $\mathcal{K}$ is the integral operator. Usually a linear functional from the solution:

$$
J(u)=\int \varphi(x) u(x) d x=(\varphi, u)
$$

The work is supported by KP-06-M32/2-17.12.2019 'Advanced Stochastic and Deterministic Approaches for Large-Scale Problems of Computational Mathematics" and by the National Scientific Program "Information and Communication Technologies for a Single Digital Market in Science, Education and Security" (ICTinSES), contract No. D01-205/23.11.2018, financed by the Ministry of Education and Science. The work is supported by the Bulgarian National Science Fund under Project DN 12/5-2017 "Efficient Stochastic Methods and Algorithms for Large-Scale Problems" and by the Project KP-06-Russia/17 "New Highly Efficient Stochastic Simulation Methods and Applications" funded by the National Science Fund - Bulgaria. should be evaluated in various problems. A MC algorithm is described below. Let $\varphi(x) \in L_{2}(\Omega)$. A set of permissible densities is defined:

$$
\begin{gathered}
\pi(x), p\left(x, x^{\prime}\right): \pi(x) \geq 0, p\left(x, x^{\prime}\right) \geq 0, \\
\int_{\Omega} \pi(x) d x=1, \int_{\Omega} p\left(x, x^{\prime}\right) d x^{\prime}=1, x \in \Omega \subset \mathbb{R}^{d} .
\end{gathered}
$$

We define a Markov chain $T_{k}: x_{0} \rightarrow x_{1} \rightarrow \cdots \rightarrow x_{k}$ [4] with length $k$ started from the initial state $x_{0}$. If the approximate initial solution coincides with the corresponding right-hand side $f(x)$, a MC algorithm for integral equations [6] is defined by:

$$
\begin{gathered}
E \theta_{k}[\varphi]=\left(\varphi, u^{(k)}\right), \theta_{k}[\varphi]=\frac{\varphi\left(x_{0}\right)}{\pi\left(x_{0}\right)} \sum_{j=0}^{k} W_{j} f\left(x_{j}\right), \\
W_{0}=1, W_{j}=W_{j-1} \frac{k\left(x_{j-1}, x_{j}\right)}{p\left(x_{j-1}, x_{j}\right)}, j=1, \ldots, k, \\
\left(\varphi, u^{(k)}\right) \approx \frac{1}{N} \sum_{n=1}^{N} \theta_{k}[\varphi]_{n} .
\end{gathered}
$$

\section{A PROBABILISTIC ERROR ESTIMATE}

The probabilistic error is $r_{N} \leq 0.6745 \sigma(\theta) \frac{1}{\sqrt{N}}$ [3], [2], where $N$ is the number of samples of the random variable $\theta$ and $\sigma(\theta)=(D \theta)^{1 / 2}$ is the standard deviation of the random variable $\theta$ for which $E \theta_{k}[\varphi]=\left(\varphi, u^{(k)}\right)=\sum_{j=0}^{k}\left(\varphi, \mathcal{K}^{(j)} f\right)$, where for point $x=\left(x_{0}, \ldots, x_{j}\right) \in G \equiv \Omega^{j+1} \subset$ $\mathbb{R}^{d(j+1)}, j=1, \ldots, k$ :

$$
\begin{gathered}
\left(\varphi, \mathcal{K}^{(j)} f\right)=\int_{\Omega} \varphi\left(x_{0}\right) \mathcal{K}^{(j)} f\left(x_{0}\right) d x_{0}= \\
=\int_{G} \varphi\left(x_{0}\right) k\left(x_{0}, x_{1}\right) \ldots k\left(x_{k-1}, x_{j}\right) f\left(x_{j}\right) d x_{0} d x_{1} \ldots d x_{j}=
\end{gathered}
$$




$$
\int_{G} F(x) d x
$$

where

$F(x)=\varphi\left(x_{0}\right) k\left(x_{0}, x_{1}\right) \ldots k\left(x_{k-1}, x_{j}\right) f\left(x_{j}\right), x \in G \subset \mathbb{R}^{d(j+1)}$.

Using the inequality $D \sum_{j=0}^{k} \theta_{k}^{(j)} \leq\left(\sum_{j=0}^{k} \sqrt{D \theta_{k}^{(j)}}\right)^{2}$, and the variance properties we have the following inequalities [1]:

$r_{N} \leq$

$$
\begin{aligned}
& \frac{0.6745}{\sqrt{N}} \sum_{j=0}^{k}\left(\int_{G}\left(\mathcal{K}^{(j)} \varphi f\right)^{2} p d x-\left(\int_{G} \mathcal{K}^{(j)} \varphi f p d x\right)^{2}\right)^{1 / 2} \leq \\
& \leq \frac{0.6745}{\sqrt{N}} \sum_{j=0}^{k}\left(\int_{G}\left(\mathcal{K}^{(j)} \varphi f\right)^{2} p d x\right)^{1 / 2} \\
& =\frac{0.6745}{\sqrt{N}}\|\varphi\|_{L_{2}}\|f\|_{L_{2}} \sum_{j=0}^{k}\left\|\mathcal{K}^{(j)}\right\|_{L_{2}} .
\end{aligned}
$$

The following estimate is obtained:

$$
r_{N} \leq \frac{0.6745\|f\|_{L_{2}}\|\varphi\|_{L_{2}}}{\sqrt{N}\left(1-\|\mathcal{K}\|_{L_{2}}\right)}
$$

\section{A SYSTEMATIC ERROR ESTIMATE}

The sequence [2] $u^{(1)}, u^{(2)}, \ldots$ is defined by the recursion formula $u^{(k)}=\mathcal{K} u^{(k-1)}+f, k=1,2, \ldots$ The formal solution of the equation (1) is the truncated Neumann series $u^{(k)}=f+$ $\mathcal{K} f+\cdots+\mathcal{K}^{(k-1)} f+\mathcal{K}^{(k)} u^{(0)}, k>0$, where the $k^{\text {th }}$ iteration of $\mathcal{K}$ is denoted by $\mathcal{K}^{(k)}$, and $u^{(k)}=\sum_{i=0}^{k-1} \mathcal{K}^{(i)} f+\mathcal{K}^{(k)} u^{(0)}$.

We construct the $k$ - residual vector of the systematic error $r^{(k)}: r^{(k)}=f-(I-\mathcal{K}) u^{(k)}=(I-\mathcal{K})\left(u-u^{(k)}\right)$.

By the definition of $r^{(k)}: r^{(k)}=f-u^{(k)}+\mathcal{K} u^{(k)}=$ $u^{(k+1)}-u^{(k)}$ and $r^{(k+1)}=u^{(k+2)}-u^{(k+1)}=\mathcal{K} u^{(k+1)}+f-$ $\mathcal{K} u^{(k)}-f=\mathcal{K}\left(u^{(k+1)}-u^{(k)}\right)=\mathcal{K} r^{(k)}$.

We have $r^{(0)}=u^{(1)}-u^{(0)}=\mathcal{K} u^{(0)}+f-u^{(0)}=\mathcal{K} f$, $r^{(k+1)}=\mathcal{K} r^{(k)}=\mathcal{K}^{(2)} r^{(k-1)}=\cdots=\mathcal{K}^{(k+1)} r^{(0)}$.

So we obtain $u^{(k+1)}=u^{(k)}+r^{(k)}=u^{(k-1)}+r^{(k-1)}+$ $r^{(k)}=\cdots=u^{(0)}+r^{(0)}+\cdots+r^{(k)}=u^{(0)}+r^{(0)}+\mathcal{K} r^{(0)}+$ $\mathcal{K}^{(2)} r^{(0)}+\cdots+\mathcal{K}^{(k)} r^{(0)}=u^{(0)}+\left(I+\mathcal{K}+\cdots+\mathcal{K}^{(k)}\right) r^{(0)}$ [4].

If $\|\mathcal{K}\|_{L_{2}}<1$ then the Neumann series $u=\sum_{i=0}^{\infty} \mathcal{K}^{(i)} f$ is convergent and $u^{(k+1)} \stackrel{k \rightarrow \infty}{\longrightarrow} u$ therefore from $u^{(k+1)}=$ $u^{(0)}+\left(I+\mathcal{K}+\cdots+\mathcal{K}^{(k)}\right) r^{(0)}$ and $k \rightarrow \infty$ we have $u=$ $u^{(0)}+(I-\mathcal{K})^{-1} r^{(0)}$. After simple transformations $u=\mathcal{K} u+$ $f=\mathcal{K} u^{(0)}+\mathcal{K}(I-\mathcal{K})^{-1} r^{(0)}+f=u^{(1)}+\mathcal{K}(I-\mathcal{K})^{-1} r^{(0)}$.

Doing this $k$ times we obtain: $u=u^{(k)}+$ $\mathcal{K}^{(k)}(I-\mathcal{K})^{-1} r^{(0)}$. The following inequalities are established applying the Cauchy-Schwarz inequality:

$$
r^{(k)}=\left\|u-u^{(k)}\right\|_{L_{2}} \leq
$$

$$
\frac{\|\mathcal{K}\|_{L_{2}}^{k}\left\|r^{(0)}\right\|_{L_{2}}}{1-\|\mathcal{K}\|_{L_{2}}} \leq \frac{\|\mathcal{K}\|_{L_{2}}^{k}\|f\|_{L_{2}}\|\mathcal{K}\|_{L_{2}}}{1-\|\mathcal{K}\|_{L_{2}}}=
$$

$$
\frac{\|\mathcal{K}\|_{L_{2}}^{k+1}\|f\|_{L_{2}}}{1-\|\mathcal{K}\|_{L_{2}}}
$$

The systematic error is estimated in following way:

$$
\begin{gathered}
\left|(\varphi, u)-\left(\varphi, u^{(k)}\right)\right| \leq\|\varphi\|_{L_{2}}\left\|u-u^{(k)}\right\|_{L_{2}} \leq \\
\frac{\|\varphi\|_{L_{2}}\|f\|_{L_{2}}\|\mathcal{K}\|_{L_{2}}^{k+1}}{1-\|\mathcal{K}\|_{L_{2}}} .
\end{gathered}
$$

\section{THE OPTIMIZED STOCHASTIC APPROACH}

Let us denote by $\delta$ an accuracy to solve the task under consideration (2). This means that:

$$
r_{N} \leq \frac{0.6745\|\varphi\|_{L_{2}}\|f\|_{L_{2}}}{\sqrt{N}\left(1-\|\mathcal{K}\|_{L_{2}}\right)} \leq \frac{\delta}{2}
$$

$$
r_{k} \leq \frac{\|\varphi\|_{L_{2}}\|f\|_{L_{2}}\|\mathcal{K}\|_{L_{2}}^{k+1}}{1-\|\mathcal{K}\|_{L_{2}}} \leq \frac{\delta}{2}
$$

For a Fredholm integral equation (1) the lower bounds for $N$ and $k$ for the OPTIMIZED MC algorithm are:

$$
N \geq\left(\frac{1.349\|\varphi\|_{L_{2}}\|f\|_{L_{2}}}{\delta\left(1-\|\mathcal{K}\|_{L_{2}}\right)}\right)^{2}, k \geq \frac{\ln \frac{\delta\left(1-\|\mathcal{K}\|_{L_{2}}\right)}{2\|\varphi\|_{L_{2}}\|f\|_{L_{2}}\|\mathcal{K}\|_{L_{2}}}}{\ln \|\mathcal{K}\|_{L_{2}}} .
$$

We have also obtained an optimal ratio between $k$ and $N$ : For a Fredholm integral equation (1) the lower bounds for $N$ and $k$ for the OPTIMIZED MC algorithm are:

$$
N \geq\left(\frac{1.349\|\varphi\|_{L_{2}}\|f\|_{L_{2}}}{\delta\left(1-\|\mathcal{K}\|_{L_{2}}\right)}\right)^{2}, k \geq \frac{\ln \frac{0.6745}{\|\mathcal{K}\|_{L_{2}} \sqrt{N}}}{\ln \|\mathcal{K}\|_{L_{2}}} .
$$

\section{NUMERICAL EXAMPLES AND RESULTS}

\section{A. Example 1}

The first example is:

$$
u(x)=\int_{\Omega} k\left(x, x^{\prime}\right) u\left(x^{\prime}\right) d x^{\prime}+f(x),
$$

$\Omega \equiv[0,1], k\left(x, x^{\prime}\right)=\frac{1}{6} e^{x+x^{\prime}}, f(x)=6 x-e^{x} \cdot \varphi(x)$ is the delta function $(\Delta(x))$. The exact solution is $u(x)=6 x$. We are interested in the value of the solution at the middle of the interval. Firstly, the $L_{2}$ norms are computed: $\|\varphi\|_{L_{2}}=1$, $\|\mathcal{K}\|_{L_{2}}=0.5324,\|f\|_{L_{2}}=1.7873$. For this example the exact solution is 3 and $\pi(x)=\Delta(x)$. We make 20 algorithm runs on Intel Core i5-2410M @ $2.3 \mathrm{GHz}$. 
TABLE I

RESULTS FOR THE FIRST EXAMPLE.

\begin{tabular}{c|c|c|c|c|c|c|c}
\hline \hline$\delta$ & $\mathrm{N}$ & $\mathrm{k}$ & $\begin{array}{c}\text { expected } \\
\text { rel. error }\end{array}$ & $\begin{array}{c}\text { BASIC } \\
\text { rel. error }\end{array}$ & $\begin{array}{c}\text { time } \\
\text { (sec.) }\end{array}$ & $\begin{array}{c}\text { OPTIMIZED } \\
\text { rel. error }\end{array}$ & $\begin{array}{c}\text { time } \\
\text { (sec.) }\end{array}$ \\
\hline \hline 0.1 & 2659 & 6 & 0.0333 & 0.0137 & 11 & 0.0132 & 5 \\
\hline 0.03 & 29542 & 8 & 0.01 & 0.0039 & 62 & 0.0036 & 42 \\
\hline 0.02 & 66468 & 9 & 0.0067 & 0.0022 & 140 & 0.0020 & 70 \\
\hline 0.0075 & 472659 & 10 & 0.0025 & 0.001 & 1167 & $9.3671 \mathrm{e}-04$ & 529 \\
\hline 0.007 & 542593 & 11 & 0.00233 & $6.9639 \mathrm{e}-04$ & 1562 & $6.3582 \mathrm{e}-04$ & 614 \\
\hline 0.005 & 1063482 & 11 & 0.00167 & $6.4221 \mathrm{e}-04$ & 4412 & $6.2479 \mathrm{e}-04$ & 2202 \\
\hline
\end{tabular}

Fig. 1. Experimental and expected relative error.

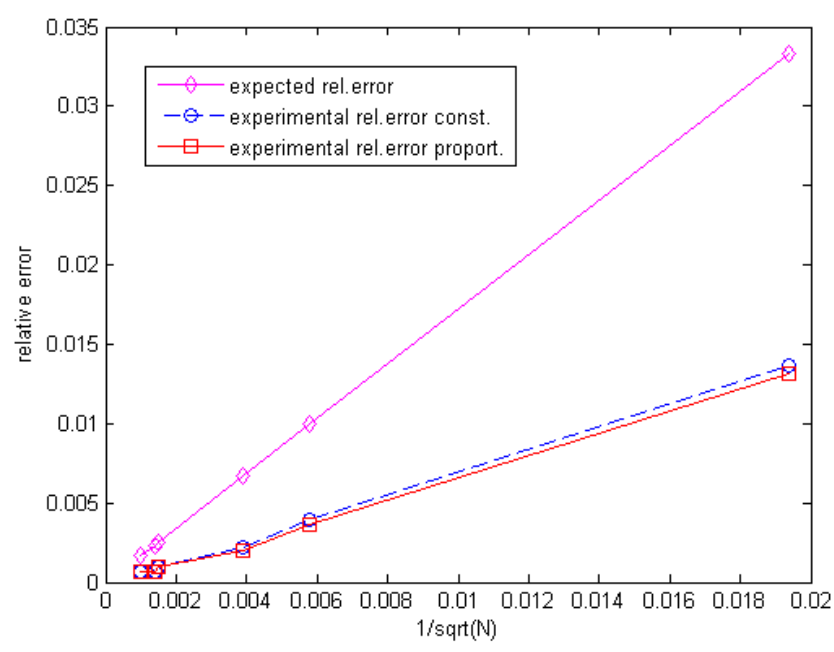

\section{B. Numerical results for the first example}

The first two columns with the expected relative error (RE) and the computational time (CPU time) measured in seconds are for the case when the transition probabilities are constant functions (this is the standard MC method and we use the notation BASIC) and the last two columns are for the case when OPTIMIZED is used (this is also called the almost optimal MC algorithm). From the Tables it leads that the OPTIMIZED method gives better results (smaller relative errors and significantly smaller computational times).

We can see the comparison between the expected and experimental relative error on Figure 1 which shows that experimental RE confirms the expected RE.

The OPTIMIZED MC algorithm has a higher computational efficiency than the BASIC MC algorithm because its CPU time is smaller.

\section{Example 2}

The next example is a biology analytically tractable model [5]:
TABLE II

RESULTS FOR THE SECOND EXAMPLE.

\begin{tabular}{c|c|c|c|c|c|c|c}
\hline \hline$\delta$ & $\mathrm{N}$ & $\mathrm{k}$ & $\begin{array}{c}\text { expected } \\
\text { rel. error }\end{array}$ & $\begin{array}{c}\text { BASIC } \\
\text { rel. error }\end{array}$ & $\begin{array}{c}\text { time } \\
\text { sec. }\end{array}$ & $\begin{array}{c}\text { OPTIMIZED } \\
\text { rel. error }\end{array}$ & time \\
sec.
\end{tabular}

$$
u(x)=\int_{\Omega} k\left(x, x^{\prime}\right) u\left(x^{\prime}\right) d x^{\prime}+f(x),
$$

$\Omega \equiv[0,1], k\left(x, x^{\prime}\right)=\frac{1}{3} e^{x}, f(x)=\frac{2}{3} e^{x} \cdot \varphi(x)$ is the delta function. The exact solution is $u(x)=e^{x}$. We are interested in the value of the solution at the middle point of the interval. The $L_{2}$ norms are evaluated as follows: $\|\varphi\|_{L_{2}}=1,\|\mathcal{K}\|_{L_{2}}=$ $0.3917,\|f\|_{L_{2}}=1.1915$. Here the exact solution is 1.6487 and $\pi(x)=\Delta(x)$. We make 20 algorithm runs on the same computational unit.

\section{Numerical results for the second example}

One can see that the OPTIMIZED method gives slightly better results than the BASIC MC and the results are closer when the initial probability is the delta function. However again the OPTIMIZED MC algorithm has a higher computational efficiency than the BASIC algorithm because its CPU time is shorter.

\section{E. Example 3}

We study the following example describes the procedure of teaching of neural networks [4], [5]:

$$
u(x)=\int_{\Omega} k\left(x, x^{\prime}\right) u\left(x^{\prime}\right) d x^{\prime}+f(x),
$$

$\Omega \equiv[-2,2], k\left(x, x^{\prime}\right)=\frac{0.055}{1+e^{-3 x}}+0.07, \quad f(x)=$ $0.02\left(3 x^{2}+e^{-0.35 x}\right), \varphi(x)=0.7\left((x+1)^{2} \cos (5 x)+20\right)$.

Here $\varphi(x)=0.7\left((x+1)^{2} \cos 5 x+20\right)$. The exact solution is 8.98635750518 [2]. We calculate: $\|\varphi\|_{L_{2}}=27.7782$, $\|\mathcal{K}\|_{L_{2}}=0.2001,\|f\|_{L_{2}}=0.2510$.

We make 20 algorithm runs on the same processor.

\section{F. Numerical results for the third example}

The results presented in Table III demonstrates that the OPTIMIZED MC method gives much smaller relative errors than the BASIC MC algorithm for larger values of $N$ and $k$. In the case of smaller values of these quantities the BASIC MC gives smaller relative errors, but the RE obtained with OPTIMIZED method are closer to the expected RE. Using the OPTIMIZED approach we see that the experimental RE confirms the expected RE. We also see that in the OPTIMIZED algorithm is a little bit slower because we use the acceptancerejection method for modeling the initial probabilities. 
TABLE III

RESULTS FOR THE THIRD EXAMPLE.

\begin{tabular}{c|c|c|c|c|c|c|c}
\hline \hline$\delta$ & $\mathrm{N}$ & $\mathrm{k}$ & $\begin{array}{c}\text { expected } \\
\text { rel. error }\end{array}$ & $\begin{array}{c}\text { BASIC } \\
\text { rel. error }\end{array}$ & $\begin{array}{c}\text { time } \\
\text { sec. }\end{array}$ & $\begin{array}{c}\text { OPTIMIZED } \\
\text { rel. error }\end{array}$ & $\begin{array}{c}\text { time } \\
\text { sec. }\end{array}$ \\
\hline 0.4 & 865 & 3 & 0.0445 & 0.0052 & 3 & 0.0239 & 5 \\
\hline 0.2 & 3457 & 4 & 0.0223 & 0.0094 & 9 & 0.0121 & 23 \\
\hline 0.1 & 13827 & 4 & 0.0111 & 0.0113 & 28 & 0.0086 & 46 \\
\hline 0.05 & 55306 & 5 & 0.00556 & 0.0177 & 132 & 0.0032 & 222 \\
\hline 0.028 & 176357 & 5 & 0.00312 & 0.0176 & 448 & 0.0031 & 540 \\
\hline 0.02 & 345659 & 6 & 0.00233 & 0.0202 & 901 & 0.0013 & 1090 \\
\hline
\end{tabular}

\section{CONCLUSION}

In this paper we present an optimized stochastic algorithm for solving the Fredholm integral equation of the second kind. Two main cases are taken into account - the initial probability coincides with the delta function, and the second case when the initial probability is different from the delta function. The results from the numerical tests in the first case show that the OPTIMIZED MC reaches much smaller computational times than the BASIC MC with constant probabilities and comparable relative errors respectively. The results from the numerical tests in the second case show that the OPTIMIZED MC reaches much smaller relative errors than the BASIC MC with constant probabilities and comparable computational times respectively. the OPTIMIZED MC has a higher computational efficiency than the BASIC MC. The main conclusion here is that the OPTIMIZED MC approach is characterized by a higher computational efficiency (proportional to relative error and compuatational time) in both cases under consideration.

\section{REFERENCES}

[1] I. Dimov, Monte Carlo Methods for Applied Scientists, New Jersey, London, Singapore, World Scientific, 2008, 291p.

[2] R. Georgieva, PhD Thesis: Computational complexity of Monte Carlo algorithms for multidimensional integrals and integral equations, Sofia, 2003

[3] I. Dimov, E. Atanassov, What Monte Carlo models can do and cannot do efficiently?, Applied Mathematical Modelling 32 (2007) 1477-1500.

[4] J.H. Curtiss. Monte Carlo Methods for The Iteration of Linear Operators. J. Math. Phys., 32 209-232, (1954)

[5] A. Doucet, A.M. Johansen, V.B. Tadic. On solving integral equations using Markov chain Monte Carlo methods. Applied Mathematics and Computations, 216 2869-2880, (2010).

[6] I. Sobol. Numerical methods Monte Carlo. Nauka, Moscow, 1973.

[7] S. L. Zaharieva, I. Radoslavov Georgiev, V. A. Mutkov and Y. Branimirov Neikov, "Arima Approach For Forecasting Temperature In A Residential Premises Part 2," 2021 20th International Symposium Infoteh-jahorina (infoteh), 2021, pp. 1-5. 